\title{
Crescimento de novilhas de corte com diferentes ganhos de peso dos 12 aos 18 meses de idade
}

\author{
Beef heifers growth with different live weight gain from 12 to 18 months of age \\ Eduardo Castro da Costa, Júlio Otávio Jardim Barcellos, Vanessa Peripolli, \\ Tamara Esteves de Oliveira, J orge López \& J osé Braccini Neto
}

\begin{abstract}
RESUMO
A recria de novilhas é uma etapa estratégica, sob o ponto de vista bioeconômico, e depende essencialmente de programas alimentares do desmame ao primeiro acasalamento ajustados aos diferentes sistemas de produção. O objetivo do experimento foi avaliar o efeito do ganho de peso (GP) sobre o crescimento de novilhas de corte nos períodos dos 12 aos 15 meses (P15) e dos 15 aos 18 meses (P18) de idade. As novilhas tinham idade média de 12 meses e peso vivo inicial de 170,5; 188,6 e 230,5 kg para os respectivos tratamentos: AM (Alto-Moderado), MA (Moderado-Alto) e BB (Baixo-Baixo), que foram diferentes sequências de GP nos períodos P15 e P18. As características avaliadas foram: escore de condição corporal (ECC, escala de 5 pontos), altura da garupa (AG), perímetro torácico (PT) e relação peso:altura (PA). Ao final do experimento, os três tratamentos convergiram no peso vivo médio de $312 \mathrm{~kg}$ e apresentaram valores semelhantes para AG, PT e relação peso:altura (P> 0,05). O tratamento AM que apresentou menor GP no P18 que no P15, perdeu 0,38 pontos de ECC, resultando em menor escore final que nos tratamentos MA e BB $(\mathrm{P}<0,05)$. O ganho de AG mostrou pouca relação com o GP. O efeito do ganho de peso sobre o ganho de PT ocorreu somente no P15, em que a taxa de crescimento foi maior. O ganho de PA respondeu linearmente em todos os tratamentos ao GP e foram altamente correlacionados.
\end{abstract}

Descritores: altura da garupa, bovinos de corte, escore de condição corporal, perímetro torácico, relação peso:altura.

\section{ABSTRACT}

The dairy heifer is a strategic step in terms of bioeconomic and depends primarily on food programs of weaning the first mating adjusted to the different production systems. The purpose of the experiment was to evaluate the effect of live weight gain on beef heifers growth during the periods from 12 to 15 months of age (P15) and from 15 to 18 months of age (P18). The heifers were around 12 months old at the beginning of the trial and weighted $170.2,188.6$ e $230.5 \mathrm{~kg}$ in the respective treatments: HM (High-Moderate), MH (Moderate-High) and LL (Low-Low), that were different live weight gains sequences on P15 and P18 periods. The parameters evaluated were hip height, hearth girth, weight:height ratio and body condition score (BCS, 5 points scale). At the end of the experiment the animals showed an average live weight of $312.05 \mathrm{~kg}$ and did not show differences for hip height, hearth girth and weight:height ratio $(\mathrm{P}>0.05)$. The treatment HM showed smaller live weight gain in the P18 than in the $\mathrm{P} 15$ period and lost 0.38 points of BCS in the $\mathrm{P} 18$ period, resulting in smaller final BCS than $\mathrm{MH}$ and LL treatments $(\mathrm{P}<$ 0.05). The hip height gain showed a trifle relation with live weight gain. The live weight gain influence on hearth girth gain was observed only in the P15 period, when the growth rate was higher. The weight:height ratio gain response to live weight gain was always linear and with a high relationship.

Keywords: beef cattle, body condition score, hearth girth, hip height, weight:height ratio. 


\section{INTRODUÇÃO}

A recria de novilhas é uma etapa estratégica, sob o ponto de vista bioeconômico, e depende essencialmente de programas alimentares do desmame ao primeiro acasalamento, ajustados aos diferentes sistemas de produção. Diferentes manejos e regimes alimentares influenciam as características de crescimento como a área pélvica e a altura de garupa [12] e as novilhas com maiores dificuldade no parto são mais leves, de menor altura e área pélvica que as demais [2]. A taxa de ganho de peso e o potencial genético, segundo a idade cronológica, são os principais fatores que controlam o fenômeno do crescimento nos diferentes tecidos [5]. Algumas características de medida corporal aos 12 meses, como a altura da garupa, podem caracterizar a adaptação ao ambiente, influenciando o seu desempenho e servindo de base para identificação precoce de vacas com fenótipo adaptado [7]. Contudo, outras medidas como a relação peso:altura e o perímetro torácico também poderão estar relacionadas à composição corporal e ao estágio de maturação fisiológica na novilha de corte [9].

$\mathrm{O}$ entendimento das relações entre o ganho de peso, o período de vida das novilhas em que ocorre esse ganho e a resposta nas características de crescimento podem estar relacionadas com o desempenho reprodutivo futuro e, são necessárias na produção eficiente de novilhas destinadas ao primeiro acasalamento aos 18 meses de idade [4].

O objetivo deste experimento foi avaliar o efeito de sequências de ganhos de peso, dos 12 aos 15 e dos 15 aos 18 meses de idade, sobre medidas de crescimento corporal de novilhas de corte, alimentadas para alcançar um mesmo peso de acasalamento, no outono, aos 18 meses de idade.

\section{MATERIAIS E MÉTODOS}

O experimento foi realizado no Rio Grande do Sul, no período de 16 de setembro de 2004 até 29 de março de 2005. O clima da região é classificado como Cfa, tipo subtropical, com chuvas regularmente distribuídas durante o ano, podendo ocorrer períodos de estiagem nos meses de janeiro e fevereiro. A precipitação pluvial anual média é de $1350 \mathrm{~mm}$ e a temperatura anual média é de $17,6^{\circ} \mathrm{C}$, variando de $12,5^{\circ} \mathrm{C}$, em junho, até $24^{\circ} \mathrm{C}$, em janeiro. A pastagem nativa do local é composta por espécies de gramíneas rizomatosas e estoloníferas, com a presença de leguminosas, com maior crescimento na primavera e principalmente no verão.

Foram utilizadas 191 novilhas Hereford com idade inicial de 12 meses, originadas de um rebanho comercial, constituindo três lotes com diferentes pesos iniciais. A partir do desmame, aos sete meses de idade, foram distribuídas aleatoriamente em três lotes e submetidas a três sistemas de alimentação até os 12 meses de idade. Portanto, as diferenças de peso que as novilhas tinham neste experimento são atribuídas unicamente ao efeito do manejo alimentar na fase de desmama.

O experimento foi dividido em dois períodos: $1^{\circ}$ período (P15), dos 12 aos 15 meses de idade $\left(16 / 09\right.$ até $16 / 12=91$ dias) e $2^{\circ}$ período (P18), dos 15 aos 18 meses de idade $(17 / 12$ até 29/03 = 102 dias). Os tratamentos foram diferentes ganhos de peso (GP), de modo que os três lotes atingissem o mesmo peso final no acasalamento, aos 18 meses, pois há um pressuposto básico de que o peso no início do acasalamento é a variável mais importante para o desempenho reprodutivo nessa idade. Os sistemas alimentares foram planejados de acordo com o ganho de peso diário médio (GDM) no primeiro período (P15): Alto $(0,900 \mathrm{~kg} / \mathrm{d})$ e Moderado $(0,650 \mathrm{~kg} / \mathrm{d})$ (AM); Moderado $(0,490)$ e no segundo período (P18): Alto $(0,760)$ (MA); Baixo $(0,460)$ e Baixo $(0,480)$ (BB). A definição de alto, moderado ou baixo foi estabelecida apenas dentro do período. Isso explica que a taxa de ganho do Moderado no P15 seja semelhante ao Baixo no P18.

O peso inicial das novilhas foi de 170,5; 188,6 e $230,5 \mathrm{~kg}$ para os tratamentos AM, MA e BB, respectivamente.

A lotação foi fixa durante todo o experimento e cada grupo permaneceu, ao longo de todo o período, no mesmo piquete. O controle do ganho de peso dos animais foi feito por meio de diferentes lotações no início do experimento e de ajustes periódicos (a cada 35 dias) na quantidade de suplementação com concentrado energético-proteico (Tabela 1).

Ao início do experimento, as novilhas foram pesadas, foi atribuído o escore de condição corporal (ECC, escala de um até cinco) e avaliada a altura da garupa (AG) e perímetro torácico (PT), utilizando-se régua e fita métrica. A variável relação peso:altura (PA) foi obtida dividindo-se o valor de peso vivo pela medida da altura de garupa para cada observação. As avaliações de peso vivo e escore de condição cor- 
Tabela 1. Massa de forragem (MF), teor de matéria seca $\left(\mathrm{MS}, 60^{\circ} \mathrm{C}\right)$ verde na pastagem, carga animal (CA), lotação, nível de suplemento fornecido (percentagem do peso vivo), período de fornecimento (Dias) e quantidade consumida $(\mathrm{kg} / \mathrm{cab})$ para novilhas no $1^{\circ}$ período $(\mathrm{P} 15)$ e $2^{\circ}$ período $(\mathrm{P} 18)$.

\begin{tabular}{|c|c|c|c|c|c|c|c|}
\hline Tratamento $^{@}$ & $\begin{array}{c}\text { MF, } \\
\mathrm{kg} \text { MS/ha }\end{array}$ & $\begin{array}{l}\text { MS verde, } \\
\% \text { da MS }\end{array}$ & $\begin{array}{c}\text { CA, } \\
\mathrm{kg} \text { PV/ha }\end{array}$ & $\begin{array}{c}\text { Lotação, } \\
\text { novilha/ha }\end{array}$ & PV & Dias & $\begin{array}{l}\text { Suplem. } \\
\text { kg/cab* }\end{array}$ \\
\hline & \multicolumn{7}{|c|}{ P15 - 16/09/2004 - 16/12/2004 } \\
\hline $\mathrm{AM}$ & 1641,3 & 48,6 & 213,1 & 0,96 & 0,8 & 59 & 102,54 \\
\hline MA & 1510,4 & 45,8 & 230,8 & 1,03 & 0,7 & 32 & 64,71 \\
\hline \multirow[t]{2}{*}{$\mathrm{BB}$} & 2310,0 & 47,3 & 182,0 & 0,77 & 0,0 & 0 & 0 \\
\hline & \multicolumn{7}{|c|}{ P18 - 17/12/2004 - 29/03/2005 } \\
\hline $\mathrm{AM}$ & 1424,9 & 43,2 & 282,0 & 0,96 & 0,5 & 66 & 45,63 \\
\hline MA & 1346,9 & 44,6 & 297,7 & 1,03 & 0,5 & 36 & 58,24 \\
\hline $\mathrm{BB}$ & 1768,9 & 8,9 & 210,1 & 0,77 & 0,7 & 27 & 52,31 \\
\hline
\end{tabular}

*Suplemento como oferecido, com $11,5 \%$ de umidade e $17 \%$ de proteína bruta com base na matéria seca. ${ }^{\circledR}$ Sequência de ganho de peso em dois períodos (12-15 e 15-18 meses de idade) em que: $\mathrm{B}=$ baixo ganho de peso; $\mathrm{M}=$ ganho de peso moderado; $\mathrm{A}=$ alto ganho de peso.

poral foram realizadas a cada 35 dias e as medidas de altura da garupa e perímetro torácico a cada 70 dias, sempre após jejum total de 14 horas.

Foram coletadas sete amostras da pastagem de $0,25 \mathrm{~m}^{2}$, a cada 70 dias, cortadas rente ao solo em cada área de pastejo, para avaliar o teor de umidade $\left(60^{\circ} \mathrm{C}\right)$, separação da fração verde (MSv) da fração morta e senescente e estimativa da massa de forragem (MF) disponível por hectare.

$\mathrm{O}$ experimento foi composto de três tratamentos aplicados a grupos pré-formados, com diferente número de repetições, em que a repetição foi o animal. Após a análise de normalidade dos dados, por meio do teste de Shapiro-Wilk (SAS, 1999), as variáveis com distribuição normal foram submetidas à análise da variância pelo método dos modelos lineares gerais e as médias ajustadas foram comparadas pelo teste t. As variáveis cuja distribuição não se ajustou à curva normal foram comparadas pelo teste de Kruskal-Wallis (SAS, 1999). Foram feitas também a análise de correlação simples (coeficiente de Pearson ou Spearman) e regressão polinomial. Foram utilizados os procedimentos UNIVARIATE, GLM, NPAR1WAY, CORR E REG do programa estatístico SAS versão 8.02 (SAS, 1999).

\section{RESULTADOS}

Os dados de peso, medidas corporais e ECC, no início e final dos períodos, estão demonstrados na Tabela 2. A diferença de peso entre os tratamentos
AM e MA desapareceu ao final do $1^{\circ}$ período (P15) de modo que, aos 15 meses de idade, apenas existiu diferença entre o ECC dos dois tratamentos (Tabela 2). A superioridade das características das novilhas que apresentaram maior peso inicial foi mantida até o final do P15, mesmo com menor GDM neste período. Durante o P18, a taxa de crescimento das novilhas permitiu que os três grupos atingissem um peso final médio de $312 \mathrm{~kg}$. O tratamento BB teve como objetivo inicial o ganho de peso constante nas duas fases $(\mathrm{GDM}=0,430 \mathrm{~kg}$ ). Entretanto, no P15 o ganho foi superior ao esperado e desta forma, o ganho necessário no P18 para um peso final semelhante aos outros grupos foi bastante reduzido (Tabela 3 ).

A análise de regressão não mostrou efeito do ganho de peso sobre o ganho de AG no P15 em nenhum tratamento, enquanto no P18 (Tabela 4), no tratamento MA ocorreu efeito linear em que a AG aumentou $0,7 \mathrm{~cm}$ para cada $10 \mathrm{~kg}$ a mais de GP. No tratamento AM, o ganho em altura apresentou resposta quadrática em relação ao ganho de peso; todavia a variação no ganho de peso é responsável por somente $9 \%$ da variação no ganho em altura de garupa $\left(r^{2}=0,09\right)$. Na equação geral, a partir do GP de 74 $\mathrm{kg}(\mathrm{GDM}=0,728)$ entre os 15 e os 18 meses, o aumento do GP não resultou em aumento de ganho da altura da garupa, enquanto no tratamento AM, esse ponto foi encontrado quando o GDM foi de $0,469 \mathrm{~kg} /$ dia.

A relação peso:altura (PA) é uma medida que demonstra a harmonia estrutural do crescimento e 
Tabela 2. Estatísticas das características de novilhas avaliadas aos 12,15 e 18 meses de idade.

\begin{tabular}{|c|c|c|c|}
\hline \multirow[b]{2}{*}{ Característica } & \multicolumn{3}{|c|}{ Tratamento $^{@}$} \\
\hline & $\mathrm{AM}(\mathrm{n}=70)$ & MA $(n=61)$ & $\mathrm{BB}(\mathrm{n}=60)$ \\
\hline & \multicolumn{3}{|c|}{12 meses de idade - 16/09/2004 } \\
\hline Peso, $\mathrm{kg}^{\S}$ & $170,5 \pm 2,27 \mathrm{C}$ & $188,6 \pm 2,43 B$ & $230,5 \pm 2,45 \mathrm{~A}$ \\
\hline Altura da garupa, $\mathrm{cm}^{\S}$ & $107,7 \pm 0,39 \mathrm{~B}$ & $107,9 \pm 0,42 B$ & $111,5 \pm 0,43 \mathrm{C}$ \\
\hline Perímetro torácico, $\mathrm{cm}^{\S}$ & $134,4 \pm 0,58 \mathrm{C}$ & $137,1 \pm 0,62 B$ & $145,5 \pm 0,63 \mathrm{C}$ \\
\hline Relação peso:altura, $\mathrm{kg} / \mathrm{cm}^{\S}$ & $1,58 \pm 0,01 \mathrm{C}$ & $1,74 \pm 0,01 \mathrm{~B}$ & $2,06 \pm 0,01 \mathrm{~A}$ \\
\hline Condição corporal $^{* \#} \quad \mathrm{SD}=0,32$ & $2,5 \mathrm{C}$ & $2,7 \mathrm{~B}$ & $3,0 \mathrm{~A}$ \\
\hline & \multicolumn{3}{|c|}{15 meses de idade $-17 / 12 / 2004$} \\
\hline Peso, $\mathrm{kg}^{\S}$ & $265,6 \pm 2,67 \mathrm{~B}$ & $260,4 \pm 2,86 \mathrm{~B}$ & $281,0 \pm 2,89 \mathrm{~A}$ \\
\hline Altura da garupa, $\mathrm{cm}^{\S}$ & $115,7 \pm 0,38 \mathrm{~B}$ & $115,5 \pm 0,41 \mathrm{~B}$ & $119,0 \pm 0,41 \mathrm{~A}$ \\
\hline Perímetro torácico, $\mathrm{cm}^{\S}$ & $152,5 \pm 0,58 \mathrm{~B}$ & $151,9 \pm 0,62 \mathrm{~B}$ & $154,8 \pm 0,63 \mathrm{~A}$ \\
\hline Relação peso:altura, $\mathrm{kg} / \mathrm{cm}^{\S}$ & $2,29 \pm 0,01 B$ & $2,25 \pm 0,02 \mathrm{~B}$ & $2,36 \pm 0,02 \mathrm{~A}$ \\
\hline Condição corporal $^{* \#} \mid \mathrm{SD}=0,38$ & $3,5 \mathrm{~A}$ & $3,4 \mathrm{~B}$ & $3,6 \mathrm{~A}$ \\
\hline & \multicolumn{3}{|c|}{18 meses de idade $-29 / 03 / 2005$} \\
\hline Peso, $\mathrm{kg}^{\S}$ & $312,3 \pm 3,26$ & $314,7 \pm 3,50$ & $309,0 \pm 3,53$ \\
\hline Altura da garupa, $\mathrm{cm}^{\S}$ & $120,3 \pm 0,39$ & $120,0 \pm 0,42$ & $121,7 \pm 0,42$ \\
\hline Perímetro torácico, $\mathrm{cm}^{\S}$ & $160,4 \pm 0,60$ & $160,7 \pm 0,64$ & $162,1 \pm 0,65$ \\
\hline Relação peso:altura, $\mathrm{kg} / \mathrm{cm}^{\S}$ & $2,59 \pm 0,02$ & $2,62 \pm 0,02$ & $2,53 \pm 0,02$ \\
\hline Condição corporal $^{* \#} \quad \mathrm{SD}=0,50$ & $3,2 \mathrm{~B}$ & $3,7 \mathrm{~A}$ & $3,6 \mathrm{~A}$ \\
\hline
\end{tabular}

*Escala de 1 a 5, em que 1 significa animal muito magro e 5 significa muito gordo. Médias na mesma linha seguidas por letras diferentes são diferentes $(\mathrm{P}<0,05) .{ }^{\S}$ - médias estimadas pelo método dos quadrados mínimos e comparadas pelo teste t.*-Comparações pelo teste de Kruskal-Wallis. ${ }^{\circledR}$ Sequência de ganho de peso em dois períodos (12-15 e 15-18 meses de idade) em que: $\mathrm{B}=$ baixo ganho de peso; $\mathrm{M}=$ ganho de peso moderado; $\mathrm{A}=$ alto ganho de peso.

que não depende do tamanho adulto, pois novilhas de diferentes potenciais de crescimento atingiram a puberdade com pesos e alturas diferentes, mas com uma mesma relação PA [6]. O ganho em PA teve resposta linear ao ganho de peso, tendo maior magnitude no P15, em que o ganho de peso foi mais elevado (Tabela 4). O ganho de PA sempre foi altamente correlacionado com o GP ( $r=0,86$ a r= 0,$95 ; \mathrm{P}<0,0001)$; todavia, a correlação com o ganho de AG foi inversa no grupo AM nas duas fases, assim como no tratamento MA durante o P15, mas não no P18.

As novilhas apresentaram distintas respostas do ganho de PT frente ao ganho de peso no P15, conforme o tratamento a que foram submetidas (Tabela 4).

$\mathrm{O}$ tratamento AM respondeu linearmente ao aumento de ganho de peso, resultando em maior ganho de PT no P15. As novilhas do grupo MA responderam de forma quadrática, apresentando máximo ganho de 17,22 cm de PT quando ganharam 96,6 kg de peso no período; a partir desse ponto, o ganho em PT foi decrescente. Ao contrário, as novilhas do grupo BB apresentaram resposta levemente decrescente até o ganho de $41,1 \mathrm{~kg}$ de peso vivo.

No tratamento AM, o escore de condição corporal (Tabela 3) final foi inferior aos demais.

\section{DISCUSSÃO}

$\mathrm{O}$ alto peso inicial das novilhas do tratamento BB permitiu que, com um GP baixo dos 12 aos 15 meses e dos 15 aos 18 meses, as novilhas atingissem peso ao acasalamento aos 18 meses superior a $300 \mathrm{~kg}$, resultados semelhantes aos obtidos por outros autores [9], que trabalharam com novilhas do mesmo rebanho para assegurar elevadas taxas de prenhez. 
No período dos 12 aos 15 meses de idade (P15), os tratamentos não diferiram quanto ao ganho em altura (Tabela 3), semelhantemente ao que ocorreu no experimento com novilhas Hereford com pesos iniciais de 210, 195 e $175 \mathrm{~kg}$, a GDM de 0,595; 0,637 e $0,723 \mathrm{~kg}$, respectivamente [9].

No P18, o ganho em altura parece ter sido limitado pelas condições de alimentação fornecidas aos animais do tratamento BB para obter o ganho de peso de 27,96 kg (GDM de 0,274 kg). Outra possibilidade é a observação de que essas novilhas eram mais altas no início desse período e já se encontravam em um estágio de desenvolvimento com menor crescimento de AG, embora ainda aquém da AG na maturidade. A intensidade do crescimento em altura da garupa foi decrescente desde os nove meses de idade. Em novilhas das raças Polled Hereford, Aberdeen Angus e Simental, o limite do crescimento em altura foi obtido entre os 24 e 27 meses e nas novilhas Polled Hereford, Aberdeen Angus e Santa Gertrudis, que cresceram em outra localidade, mais lentamente, esse limite não foi encontrado até os 39 meses de idade [12].
Os resultados da tabela 4 indicam que a altura de garupa é influenciada pelo ganho de peso até uma determinada taxa de ganho de peso, que neste experimento foi em torno de $0,5 \mathrm{~kg} / \mathrm{dia}$, e, a partir desse ponto, a altura de garupa é influenciada por outros fatores. Maior altura de cernelha, maior peso vivo e maior perímetro torácico para novilhas expostas a um maior fotoperíodo foi demonstrado por [13]. Por outro lado, alta herdabilidade para a característica altura de garupa foi encontrada [8], significando que esta medida sofre pouco efeito do meio.

A correlação inversa do ganho de AG com ganho de PA indica um crescimento proporcionalmente maior da AG que do peso, verificado nas novilhas que apresentaram menor altura da garupa inicialmente $\mathrm{e}$ desenvolveram maior crescimento quando tiveram acesso a um melhor nível alimentar (AM e MA).

A correlação entre ganho de PT e ganho de ECC em geral não foi significativa, porém, quando significativa, foi menor que a obtida entre ganho de ECC e o ganho na relação PA. Mesmo tratando-se de vacas adultas, a PA é uma adequada medida estimada

Tabela 3. Estatísticas das características de novilhas avaliadas dos 12 aos 15 (P15) e dos 15 aos 18 meses de idade (P18).

\begin{tabular}{|c|c|c|c|c|}
\hline \multirow{2}{*}{\multicolumn{2}{|c|}{ Característica }} & \multicolumn{3}{|c|}{ Tratamento $^{@}$} \\
\hline & & $\mathrm{AM}(\mathrm{n}=70)$ & MA $(n=61)$ & $\mathrm{BB}(\mathrm{n}=60)$ \\
\hline & & \multicolumn{3}{|c|}{$\mathrm{P} 15$ - 16/09/2004 - 17/12/2004 } \\
\hline \multicolumn{2}{|l|}{ Altura da garupa, $\mathrm{cm}^{\S}$} & $8,0 \pm 0,30$ & $7,6 \pm 0,32$ & $7,5 \pm 0,32$ \\
\hline \multicolumn{2}{|c|}{ Perímetro torácico, $\mathrm{cm}^{\S}$} & $18,1 \pm 0,43 \mathrm{~A}$ & $14,8 \pm 0,46 \mathrm{~B}$ & $9,3 \pm 0,46 \mathrm{C}$ \\
\hline \multicolumn{2}{|c|}{ Relação peso:altura, $\mathrm{kg} / \mathrm{cm}^{\S}$} & $0,71 \pm 0,01 \mathrm{~A}$ & $0,50 \pm 0,01 \mathrm{~B}$ & $0,29 \pm 0,01 \mathrm{C}$ \\
\hline \multicolumn{2}{|l|}{ Peso, $\mathrm{kg}^{\S}$} & $95,1 \pm 1,66 \mathrm{~A}$ & $71,8 \pm 1,77 \mathrm{~B}$ & $50,5 \pm 1,79 \mathrm{C}$ \\
\hline Condição corporal ${ }^{* \#}$ & $\mathrm{SD}=0,44$ & $1,05 \mathrm{~A}$ & $0,62 \mathrm{~B}$ & $0,54 \mathrm{~B}$ \\
\hline \multirow[t]{2}{*}{ GDM, $\mathrm{kg}^{\#}$} & $\mathrm{SD}=0,250$ & $1,034 \mathrm{~A}$ & $0,780 \mathrm{~B}$ & $0,549 \mathrm{C}$ \\
\hline & & \multicolumn{3}{|c|}{ P18 - 17/12/2004 - 29/03/2005 } \\
\hline \multicolumn{2}{|c|}{ Altura da garupa, $\mathrm{cm}^{\S}$} & $4,5 \pm 0,26 \mathrm{~A}$ & $4,4 \pm 0,28 \mathrm{~A}$ & $2,7 \pm 0,28 \mathrm{~B}$ \\
\hline \multicolumn{2}{|c|}{ Perímetro torácico, $\mathrm{cm}^{\S}$} & $7,9 \pm 0,43$ & $8,7 \pm 0,46$ & $7,2 \pm 0,46 \mathrm{C}$ \\
\hline \multicolumn{2}{|c|}{ Relação peso:altura, $\mathrm{kg} / \mathrm{cm}^{\S}$} & $0,30 \pm 0,01 \mathrm{~B}$ & $0,36 \pm 0,01 \mathrm{~A}$ & $0,17 \pm 0,01 \mathrm{C}$ \\
\hline \multicolumn{2}{|l|}{ Peso, $\mathrm{kg}^{\S}$} & $46,7 \pm 1,71 \mathrm{~B}$ & $54,3 \pm 1,84 \mathrm{~A}$ & $27,9 \pm 1,85 \mathrm{C}$ \\
\hline Condição corporal ${ }^{* \#}$ & $\mathrm{SD}=0,60$ & $-0,38 \mathrm{C}$ & $0,33 \mathrm{~A}$ & $-0,04 \mathrm{~B}$ \\
\hline GDM, $\mathrm{kg}^{\#}$ & $\mathrm{SD}=0,176$ & $0,457 \mathrm{~B}$ & $0,532 \mathrm{~A}$ & $0,274 \mathrm{C}$ \\
\hline
\end{tabular}

*Escala de 1 a 5, em que 1 significa animal muito magro e 5 significa muito gordo. Médias na mesma linha seguidas por letras diferentes são diferentes $(\mathrm{P}<0,05)$. ${ }^{\S}$ - médias estimadas pelo método dos quadrados mínimos e comparadas pelo teste t. ${ }^{\#-C o m p a r a c ̧ o ̃ e s ~ p e l o ~ t e s t e ~ d e ~ K r u s k a l-W a l l i s . ~}{ }^{\circledR}$ Sequência de ganho de peso em dois períodos (12-15 e 1518 meses de idade) em que: $\mathrm{B}=$ baixo ganho de peso; $\mathrm{M}=$ ganho de peso moderado; $\mathrm{A}=$ alto ganho de peso. 
Tabela 4. Parâmetros estimados para as características de crescimento de novilhas dos 12 aos 15 (P15) e dos 15 aos 18 meses de idade (P18).

\begin{tabular}{|c|c|c|c|c|c|c|}
\hline \multirow[t]{2}{*}{ Característica } & \multirow[t]{2}{*}{ Tratamento@ } & \multicolumn{3}{|c|}{ Parâmetros } & \multirow[t]{2}{*}{ Prob. } & \multirow[t]{2}{*}{$\mathrm{r}^{2}$} \\
\hline & & $\mathrm{B}_{0}$ & $\mathrm{~B}_{1}$ & $\mathrm{~B}_{2}$ & & \\
\hline \multirow{10}{*}{$\begin{array}{l}\text { Altura da } \\
\text { Gupa, } \\
\text { cm }\end{array}$} & & \multicolumn{5}{|c|}{ 12-15 meses de idade ( $\mathrm{P} 15)$} \\
\hline & $\mathrm{AM}$ & 8,07143 & & \multicolumn{3}{|c|}{ ns } \\
\hline & MA & 7,65574 & & \multicolumn{3}{|c|}{ ns } \\
\hline & $\mathrm{BB}$ & 7,53333 & & \multicolumn{3}{|c|}{ ns } \\
\hline & GERAL & 7,76963 & & \multicolumn{3}{|c|}{ ns } \\
\hline & & \multicolumn{5}{|c|}{ 15-18 meses de idade (P18) } \\
\hline & $\mathrm{AM}$ & $-2,70957$ & 0,32060 & $-0,00335$ & 0,0203 & 0,09 \\
\hline & MA & 0,47518 & 0,07301 & & $<, 0001$ & 0,31 \\
\hline & $\mathrm{BB}$ & 2,75661 & & & & \\
\hline & GERAL & $-0,02099$ & 0,13853 & $-0,00093211$ & 0,026 & 0,22 \\
\hline \multirow{10}{*}{$\begin{array}{l}\text { Relação } \\
\text { Peso: } \\
\text { altura } \\
\text { kg/cm }\end{array}$} & & \multicolumn{5}{|c|}{ 12-15 meses de idade (P15) } \\
\hline & $\mathrm{AM}$ & $-0,03226$ & 0,00782 & & $<, 0001$ & 0,81 \\
\hline & MA & $-0,12037$ & 0,00872 & & $<, 0001$ & 0,90 \\
\hline & BB & $-0,09339$ & 0,00766 & & $<, 0001$ & 0,83 \\
\hline & GERAL & $-0,13956$ & 0,00888 & & $<, 0001$ & 0,94 \\
\hline & & \multicolumn{5}{|c|}{ 15-18 meses de idade (P18) } \\
\hline & $\mathrm{AM}$ & $-0,06194$ & 0,00775 & & $<, 0001$ & 0,75 \\
\hline & MA & $-0,00109$ & 0,00680 & & $<, 0001$ & 0,90 \\
\hline & $\mathrm{BB}$ & $-0,02432$ & 0,00717 & & $<, 0001$ & 0,86 \\
\hline & GERAL & $-0,02491$ & 0,00712 & & $<, 0001$ & 0,89 \\
\hline \multirow{10}{*}{$\begin{array}{l}\text { Perímetro } \\
\text { torácico, } \\
\mathrm{cm}\end{array}$} & & \multicolumn{5}{|c|}{ 12-15 meses de idade (P15) } \\
\hline & $\mathrm{AM}$ & 5,05363 & 0,13744 & & $<, 0001$ & 0,27 \\
\hline & MA & $-10,30392$ & 0,56990 & $-0,00295$ & 0,022 & 0,47 \\
\hline & $\mathrm{BB}$ & 14,31734 & $-0,29940$ & 0,00364 & 0,0001 & 0,43 \\
\hline & GERAL & 1,24374 & 0,17740 & & $<, 0001$ & 0,63 \\
\hline & & \multicolumn{5}{|c|}{ 15-18 meses de idade (P18) } \\
\hline & $\mathrm{AM}$ & 7,95541 & & & $\mathrm{~ns}$ & \\
\hline & MA & 5,61392 & 0,05807 & & 0,0180 & 0,09 \\
\hline & $\mathrm{BB}$ & 4,76585 & 0,08988 & & 0,0202 & 0,09 \\
\hline & GERAL & 5,26000 & 0,06342 & & $<, 0001$ & 0,09 \\
\hline
\end{tabular}

${ }^{\circledR}$ Sequência de ganho de peso em dois períodos (12-15 e 15-18 meses de idade) em que: B=baixo ganho de peso; $\mathrm{M}=$ ganho de peso moderado; $\mathrm{A}=$ alto ganho de peso.

da condição corporal quando os animais têm um mesmo histórico nutricional [11], especialmente nos animais em crescimento analisados neste trabalho.

A associação entre GP e PT aumenta com o aumento da idade e a deposição de gordura, pois, na fase de crescimento, a fração do aumento do PT atribuído ao crescimento do esqueleto é pouco influenciada pelos fatores ambientais que controlam o GP [8].

O PT, assim como PA, tem maior relação com GP quando a taxa de crescimento é maior. Confirmando 
essa observação, a correlação entre GP e ganho de PT dentro de tratamentos sempre foi positiva, e no P18 foi aproximadamente metade do valor verificado no P15.

No P18 (Tabela 3), foi avaliada uma perda de 0,38 pontos, devido ao baixo GP, que foi aproximadamente 56\% menor que no P15 e modificou a composição do ganho de peso de forma a depositar menos tecido adiposo, tanto que essa mudança pôde ser detectada visualmente na avaliação do ECC. A perda de ECC em novilhas em crescimento também ocorreu quando houve menor ganho de peso na primavera que no inverno [3]. O crescimento compensatório não alterou a composição da carcaça de novilhas que passaram por restrição alimentar quando atingiram o peso final das que não sofreram restrição, mas, durante a restrição, ocorreu redução da proporção de gordura na carcaça [15]. Dos depósitos de gordura, o que mais reduziu foi o de gordura subcutânea, avaliado visualmente na atribuição do ECC.

Apesar do ganho próximo a $100 \mathrm{~kg}$ no P15 ter resultado em ganho de ECC próximo a um ponto no tratamento $\mathrm{AM}$ e o ganho de $50,5 \mathrm{~kg}$ ter refletido em ganho de 0,5 pontos no ECC no tratamento BB (Tabela 3), não é recomendável afirmar que exista uma relação linear entre ganho de peso e ganho de ECC. O resultado do período seguinte mostra que o ECC é dinâmico e depende do ECC, da taxa de crescimento e do estágio de desenvolvimento atingidos anteriormente.

\section{CONCLUSÕES}

As diferenças nas medidas corporais de novilhas de corte aos 12 meses de idade, causadas pelos diferentes ganhos de peso após o desmame, são eliminadas por meio do manejo alimentar até o acasalamento aos 18 meses.

Em novilhas de corte, a relação entre ganho de peso e ganho de escore de condição corporal é variável e depende do estágio de crescimento atingido anteriormente.

A associação do ganho de peso com a relação peso:altura ou perímetro torácico é maior em taxas de crescimento mais elevadas.

\section{REFERÊNCIAS}

1 Barcellos J.O.J., Costa E.C., Silva M.D., Semmelmann C.E.N., Montanholi Y.R., Prates E.R., Grecellé R.A., Mendes R.C., Wunsch C. \& Rosa J.R.P. 2003. Crescimento de fêmeas bovinas de corte aplicado aos sistemas de cria. Porto Alegre: Departamento de Zootecnia - UFRGS, 72p.

2 Basarab J.A., Ruttel L.M. \& Day P.A. 1993. The efficacy of predicting dystocia in yearling beef heifers: II.Using discriminant analysis. Journal of Animal Science. 71: 1372-1380.

3 Beretta V. \& Lobato J.F.P. 1998. Sistema "um ano" de produção de carne: avaliação de estratégias alternativas de alimentação hibernal de novilhas de reposição. Revista Brasileira de Zootecnia. 27: 157-163.

4 Costa E.C., López J., Barcellos J.O.J. \& Crudeli G.A. 2007. Crescimento de Novilhas de Corte dos 7 aos $11 \mathrm{Meses}$ de Idade. Archivos Latinoamericanos de Produção Animal. 2: 45-51.

5 Di Marco O.N., Barcellos J.O.J. \& Costa E.C. 2006. Crescimento de bovinos de corte. Porto Alegre: Universidade Federal do Rio Grande do Sul, 248p.

6 Hall J.B., Staigmiller R.B., Bellows R.A., Short R.E., Moseley W.M. \& Bellows S.E. 1995. Body composition and metabolic profiles associated with puberty in beef heifers. Journal of Animal Science. 73: 3409-3420.

7 Holloway J.W., Warrington B.G., Rouquette Jr F.M., Long C.R., Owens M.K. \& Baker J.F. 1992. Forage availability x heifer phenotype interactions for Brahman-Hereford $\mathrm{F}_{1}$ yearling heifers grazing humid pasture and semiarid rangeland. Journal of Animal Science. 70: 2658-2667.

8 Mayer K. 1995. Estimates of genetic parameters for mature weight of Australian beef cows and its relationship to early growth and skeletal measures. Livestock Production Science, 44: 125-137.

9 Montanholi Y.R., Barcellos J.O.J., Borges J.B., Costa E.C., Wunsh C. \& Prates E.R. 2004. Ganho de peso na recria e desempenho reprodutivo de novilhas acasaladas com sobreano. Pesquisa Agropecuária Brasileira. 39: 1253-1259.

10 Montanholi Y.R., Barcellos J.O.J. \& Costa E.C. 2008. Variação nas medidas corporais e desenvolvimento do trato reprodutivo de novilhas de corte recriadas para o acasalamento aos 18 meses de idade. Ciência Rural. 38: 185-190.

11 Nelsen T.C., Short R.E., Reynolds W.L. \& Urick J.J. 1985. Palpated and visually assigned condition scores compared with weight, height and heart girth in Hereford and crossbred cows. Journal of Animal Science. 60: 363-368.

12 Neville Jr. W.E., Mullinix Jr.B.G., Smith J.B. \& McCormick W.C. 1978. Growth patterns for pelvic dimensions and other body measuraments of beef females. Journal of Animal Science. 47: 1080-1088. 
13 Rius A.G., Connor E.E., CapucoA.V., Kendall P.E.,Auchtung-Montgomery T.L. \& Dahl G.E. 2005. Long-day photoperiod that enhances puberty does not limit body growth in Holstein heifers. Journal of Dairy Science. 88: 4356-4365.

14 SAS Institute Inc. 1999. SAS OnlineDoc, Version 8. Cary, NC: SAS Institute Inc., Disponível em: <http://v8doc.sas.com/ sashtml/>. Acessado em: $15 \mathrm{dez} .2005$.

15 Yambayamba E.S.K., Price M.A. \& Jones S.D.M. 1996. Compensatory growth carcass tissues and visceral organs in beef heifers. Livestock Prodution Science. 46: 19-32. 